\title{
Effects of Meditation on Heart Rate Stability
}

\author{
Masaki Hoshiyama ${ }^{1}$, Alan Murray ${ }^{2}$ \\ ${ }^{1}$ Department of Education, Meisei University, Tokyo, Japan, and \\ Dept. of Pediatrics, The University of Tokyo \& Affiliated Hospitals, Tokyo, Japan \\ ${ }^{2}$ School of Engineering, and Faculty of Medical Sciences, \\ Newcastle University, Newcastle upon Tyne, UK
}

\begin{abstract}
Relaxation or meditation is expected to slow the heart rate and produce more stable rate changes. However, there is little information on specific effects of meditation training. In this study we compared heart rate stability during normal and meditation conditions.

ECGs were recorded with ethical permission from normal subjects while seated, over 30-minute periods. The first set of control recordings were made with no instruction other than to remain still without talking. The subjects were then given instruction on relaxation and meditation techniques before a second similar 30-minute study period. These two recordings were made on separate days. Twelve subjects were enroled with a mean age of 36 years. Subjects of these ages were more likely to experience heart rate changes than older subjects

The mean RR interval across all subjects during the first control recordings was $837 \pm 150 \mathrm{~ms}$ (mean $\pm S D$ ) and during relaxation and meditation $846 \pm 136$, with no significant difference. In spite of no difference in mean $R R$ interval, the RR variability (average subject $S D$ ) during meditation actually increased from $53 \pm 18 \mathrm{~ms}$ to $65 \pm 24 \mathrm{~ms}$ $(p<0.05)$. In addition, it can be noted that when examining the beat-to-beat changes, the $S D$ of beat-to-beat $R R$ interval changes was less than that for the original $R R$ distribution for both the control recordings and meditation recordings (both $p<0.001$ ). This effect was more pronounced during meditation.

The expected effect of lower heart rates and lower heart rate variability during meditation did not occur, probably due to the slower and deeper breathing during meditation inducing greater changes.
\end{abstract}

\section{Introduction}

Relaxation or meditation is expected to slow the heart rate and produce more stable rate changes. However, there is little information on specific effects of meditation training. In this study we compared heart rate stability during normal and meditation conditions.

Heart rate analysis has been used in many clinical studies, including for diabetes [1], heart transplant [2,3], Alzheimer's disease and vascular dementia [4], and sleep [5], as well as for studies of multifractal characteristics [6], variation over 24 hours [7], during sleep [8,9] and Zen meditation [10-12].

For such studies, it is important to have information on stability of heart rate over extended recording periods.

We therefore in this study chose to analyse stability over 30 minute periods.

In addition, we also analysed the effect of meditation in comparison with relaxed normal breathing.

\section{Methods}

ECGs were recorded over a 30-min period, and analysed for changes in heart rate for all subjects, and the effect of meditation with controlled respiration analysed.

\subsection{Study subjects}

Twelve subjects with no known cardiac disease were enroled, and gave ethical consent. Their ages were $36 \pm 14$ years (mean \pm standard deviation).

None of the subjects exhibited any ectopic beat during the recording periods.

\subsection{Study conditions}

Subjects were given time for normal relaxation, but without any controlled conditions. After the study was explained, subjects were then asked to sit on an office chair, while a single channel ECG was recorded to a computer for offline analysis. After a few weeks, subjects were instructed briefly in Zen meditation techniques including respiratory exercise for lower abdominal muscle respiration [10], and rerecorded in similar conditions.

Subjects were asked to remain seated and still, without talking for the 30 minute recording period. 


\subsection{ECG recordings}

Single lead ECGs were obtained with three electrodes applied directly to the chest. The ECG amplifier gain was set at 1000 . The output of the amplifier was connected to an analogue-to-digital converter at a sample rate of $250 \mathrm{~Hz}$, and stored to a computer for off-line analysis (Figure 1).

\subsection{QRS detection}

ECG were filtered using time domain Savitzky-Golay filtering $[13,14]$ and each ECG beat was detected by identifying the fast response associated with each QRS as well as by coarse grained local maxima procedures, followed by a cardiologist's confirmation.

\subsection{Heart rate analysis}

The inter beat RR intervals were determined and the instantaneous inter beat heart rate calculated.

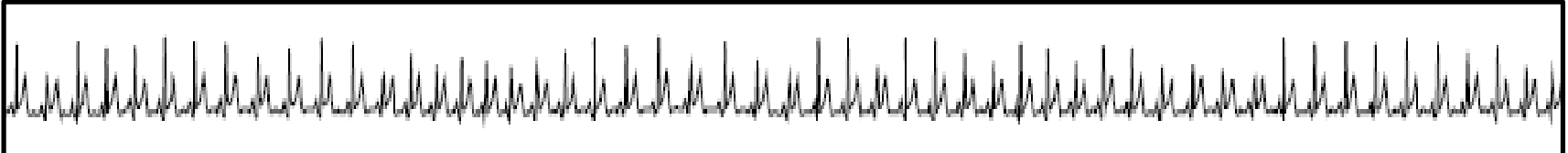

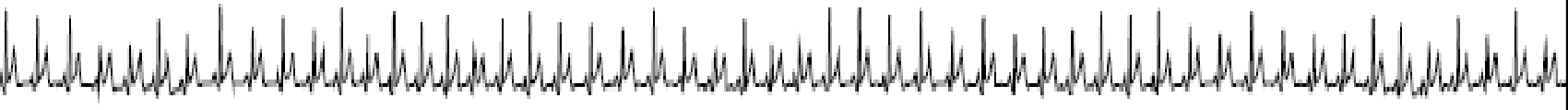

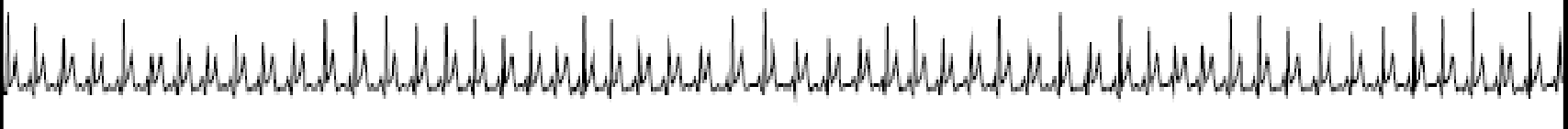

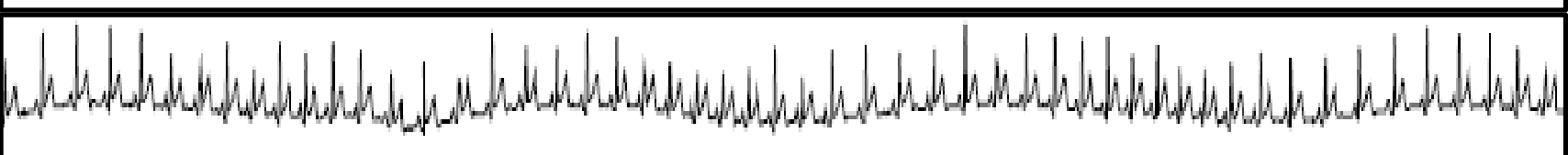



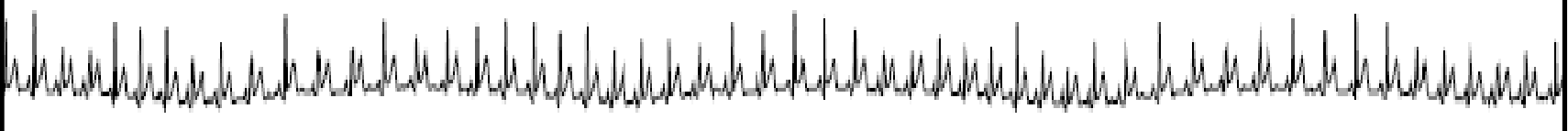

Figure 1. ECG examples from the same subject for relaxation (above) and during meditation (below). Two minutes from each 30-min recording is shown. It can be seen that regular respiratory changes are larger during meditation. 

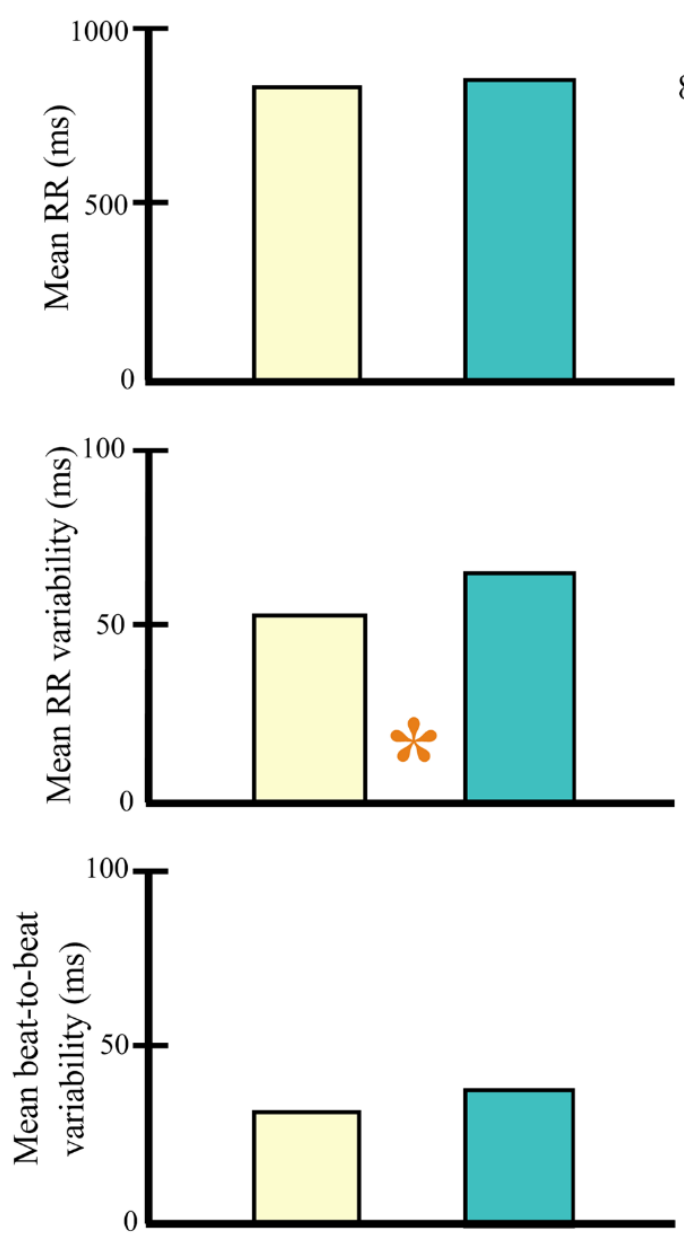

53 v $65 \mathrm{~ms}$

32 v $39 \mathrm{~ms}$



Control recordings

Relaxation / meditation recordings

$\mathrm{p}<0.05$

Figure 2. The mean RR interval across all subjects during the first control recordings (top) was $837 \pm 150 \mathrm{~ms}$ (mean $\pm \mathrm{SD}$ ) and during relaxation and meditation $846 \pm 136$, with no significant difference. The mean RR variability (average subject SD) during meditation (middle) actually increased from $53 \pm 18 \mathrm{~ms}$ to $65 \pm 24 \mathrm{~ms}(\mathrm{p}<0.05)$. There was no significant difference for mean beat-to-beat variability (bottom). 


\section{Results}

\subsection{Comparison of heart rate between control and meditation periods}

The mean RR interval across all subjects during the first control recordings was $837 \pm 150 \mathrm{~ms}$ (mean \pm SD) and during relaxation and meditation $846 \pm 136$, with no significant difference (Figure 2).

\subsection{Comparison of beat-to-beat heart rate variability between control and meditation periods}

In spite of no difference in mean RR interval, the RR variability (average subject $\mathrm{SD}$ ) during meditation actually increased from $53 \pm 18 \mathrm{~ms}$ to $65 \pm 24 \mathrm{~ms}(\mathrm{p}<0.05)$ (Figure 2).

\subsection{Comparison of variability in beat-to- beat heart rate changes between control and meditation periods}

The mean variability of beat-to-beat RR interval was less than that for the original RR distribution for both the control recordings and meditation recordings (both $\mathrm{p}<0.001)$. This effect was more pronounced during meditation (Figure 2).

\section{Discussion and conclusion}

The expected effect of lower heart rates and lower heart rate variability during meditation did not occur, probably due to the slower and deeper breathing during meditation inducing greater changes [15].

\section{Acknowledgement}

We acknowledge the support of the Great Britain Sasakawa Foundation.

\section{References}

[1] Lawrence GP, Home PD, Murray A. Repeatability of measurements and sources of variability in tests of cardiovascular autonomic function. British Heart Journal 1992;68:205-11.

[2] Lord SW, Clayton RH Mitchell L, Dark JK, Murray A, McComb JM. Sympathetic reinnervation and heart rate variability after cardiac transplantation. Heart 1997;77:5328.
[3] Lord SW, Senior RR, Das M, Whittam AM, Murray A, McComb JM. Low-frequency heart rate variability: reproducibility in cardiac transplant recipients and normal subjects. Clinical Science 2001;100:43-6.

[4] Allan LM, Kerr SRJ, Ballard CG, Allen J, Murray A, McLaren AT, Kenny RA. Autonomic function assessed by heart rate variability is normal in Alzheimer's disease and vascular dementia. Dementia and Geriatric Cognitive Disorders 2005;19:140-4.

[5] Drinnan MJ, Allen J, Langley P, Murray A. Detection of sleep apnoea from frequency analysis of heart rate variability. Computers in Cardiology 2000;27:259-62.

[6] Ramchurn SK, Murray A. Multifractal analysis of the day and night characteristics of heart rate variability. Computers in Cardiology 2002;29:421-4.

[7] Langley P, Allen J, Bowers EJ, Drinnan MJ, Haigh AJ, King ST, Olbrich T, Smith FE, Zheng D, Murray A. The ebb and flow of heart rate variability: simulation of 24 hour heart rate time series using time series data from naturally occurring phenomena. Computers in Cardiology 2005;32:973-6.

[8] Bunde A, Havlin S, Kantelhardt JW, Penzel T, Peter JH, Voigt K. Correlated and uncorrelated regions in heart-rate fluctuations during sleep. Phys Rev Lett 2000;85:3736-9.

[9] Hoshiyama M, Hoshiyama A. Heart rate variability associated with rapid eye movements during sleep. Computers in Cardiology 2007;34:689-692.

[10] Hoshiyama M, Hoshiyama A. Heart rate variability associated with different modes of lower abdominal muscle tension during Zen meditation. Computing in Cardiology 2014; 41:773-776.

[11] Hoshiyama M, Murray A. Analysis of heart rate variability indices with slowly changing heart rate. Computing in Cardiology 2016; 43:1001-1004. doi: 10.22489/CinC.2016.289-243

[12] Hoshiyama M, Murray A. Analysis in cardiac stability over thirty minute periods. Computing in Cardiology 2017;44. doi:10.22489/CinC.2017.152-183

[13] Savitzky A, Golay MJE. Smoothing and differentiation of data by simplified least squares procedures. Analytical Chemistry 1964; 36:1627-39.

[14] Steiner J, Deltour J. Smoothing and differentiation of data by simplified least square procedure. Analytical Chemistry 1972; 44:1906-9.

[15] Murray A, Hoshiyama M. Study of blood pressure during controlled respiration. Computing in Cardiology 2018;45.

Addresses for correspondence:

Professor Masaki Hoshiyama,

Department of Education, Meisei University, Tokyo, Japan M.Hoshiyama@gmail.com

Professor Alan Murray

School of Engineering, and Faculty of Medical Sciences, Newcastle University, Newcastle upon Tyne, UK.

alan.murray@ncl.ac.uk 